\title{
Formation of Pickering Emulsions Using Ion-Specific Responsive Colloids ${ }^{\dagger}$
}

\author{
Khooi Y. Tan, ${ }^{*}$ Julien E. Gautrot, ${ }^{*}$ and Wilhelm T. S. Huck*,\$, \\ ${ }^{\star}$ Melville Laboratory for Polymer Synthesis, Department of Chemistry, University of Cambridge, \\ Lensfield Road, Cambridge CB2 1 EW, United Kingdom, and ${ }^{\S}$ Radboud University Nijmegen, Institute for \\ Molecules and Materials, Heyendaalseweg 135, 6525 AJ Nijmegen, The Netherlands
}

Received July 21, 2010. Revised Manuscript Received August 27, 2010

\begin{abstract}
The ability to control the dispersion, aggregation, and assembly of colloidal systems is important for a number of applications, for instance, Pickering emulsions, drug and gene delivery, control of fluid rheology, and the formation of colloidal crystal arrays. We generated a responsive colloidal system based on polymer-brush-grafted silica nanoparticles and demonstrated that such a colloidal system can be used to produce stable oil-in-water Pickering emulsions. Cationic poly(2-(methacryloyloxy)-ethyl-trimethyl-ammonium chloride) (PMETAC) brushes were grown from silica nanoparticles (diameter $\sim 320 \mathrm{~nm}$ ) through surface-initiated atom-transfer radical polymerization (ATRP). PMETAC brushes are attractive coatings for controlling the behavior of colloidal systems, owing to their ion-specific collapse resulting in the switching of surface hydrophilicity. Fourier transform infrared (FT-IR) spectroscopy, thermogravimetric analysis (TGA), transmission electron microscopy (TEM), dynamic light scattering (DLS), and zeta-potential measurements indicated the successful grafting of PMETAC brushes on nanoparticles. The resulting colloidal dispersion was shown to be responsive to perchlorate ions $\left(\mathrm{ClO}_{4}{ }^{-}\right)$, which triggered particle aggregation and enabled the generation of Pickering emulsions. The onset of aggregation depended on the polymer chain length. Aggregation was not affected by the initiator density and brush conformational changes. Further studies suggested that particle aggregation and the formation of stable Pickering emulsions were not simply due to brush collapse but also were due to a gradual shielding of electrostatic repulsion. Finally, the stability and homogeneity of the resulting Pickering emulsions were studied
\end{abstract}

\section{Introduction}

Self-assembly and supramolecular interactions have been widely exploited to control the physical and chemical properties of surfaces and the behavior of polymer coatings. ${ }^{1}$ For example, by clicking counterions into polyelectrolyte brushes, the surface wettability can be modulated, as demonstrated by Azzaroni et al.: ${ }^{2}$ replacing $\mathrm{Cl}^{-}$ions with $\mathrm{ClO}_{4}{ }^{-}$ions significantly increased the surface hydrophobicity, showing an increase in the contact angle $\left(35\right.$ to $\left.79^{\circ}\right) \cdot \mathrm{ClO}_{4}{ }^{-}$ions interacted strongly with quaternary ammonium moieties through Coulombic forces, leading to the brush "hydrophobic-induced collapse". ${ }^{2-4}$ In addition, counterion exchange between bis(trifluoromethane) and hexametaphosphate switched the surface wettability. ${ }^{2}$ Loveless et al. ${ }^{5}$ showed that the mechanical properties of poly(4-vinylpyridine) brushes can be manipulated via bimetallic pincer-Pd ${ }^{\mathrm{II}}$ complexes that act as a supramolecular cross linker. The sequestration of counterions in polymers enables the in situ synthesis and immobilization of metallic nanoparticle catalysts. ${ }^{6-8}$ Similarly, anionic poly(3-sulfopropyl

\footnotetext{
${ }^{\dagger}$ Part of the Supramolecular Chemistry at Interfaces special issue.

*To whom correspondence should be addressed. E-mail: wtsh2@cam.ac.uk.

(1) Stuart, M. A. C. Huck, W. T. S.; Genzer, J ; Müller, M ; Ober, C. Stamm, M.; Sukhorukov, G. B.; Szleifer, I.; Tsukruk, V. V.; Urban, M.; Winnik, F.; Zauscher, S.; Luzinov, I.; Minko, S. Nat. Mater. 2010, 9, 101-113.

(2) Azzaroni, O.; Brown, A. A.; Huck, W. T. S. Adv. Mater. 2007, 19, 151-154.

(3) Azzaroni, O.; Moya, S.; Farhan, T.; Brown, A. A.; Huck, W. T. S. Macromolecules 2005, 38, 10192-10199.

(4) Lim, H. S.; Lee, S. G.; Lee, D. H.; Lee, D. Y.; Lee, S.; Cho, K. Adv. Mater 2008, 20, 4438-4441.

(5) Loveless, D. M.; Abu-Lail, N. I.; Kaholek, M.; Zauscher, S.; Craig, S. L. Angew. Chem., Int. Ed. 2006, 45, 7812-7814.

(6) Lu, Y.; Mei, Y.; Walker, R.; Ballauff, M.; Drechsler, M. Polvmer 2006, 47, 4985-4995.

(7) Li, D.; Dunlap, J. R.; Zhao, B. Langmuir 2008, 24, 5911-5918.

(8) Lu, Y.; Wittemann, A.; Ballauff, M. Macromol. Rapid Commun. 2009, 30, 806-815.

(9) Ramstedt, M.; Ekstrand-Hammarström, B.; Shchukarev, A. V.; Bucht, A.; Österlund, L.; Welch, M.; Huck, W. T. S. Biomaterials 2009, 30, 1524-1531.
}

methacrylate) brushes were loaded with silver ions and used as antibacterial coatings. ${ }^{9}$

The ability to control the surface properties and hence the dispersion, aggregation, and assembly of colloids is essential for a number of applications (e.g., biomedical diagnostics, ${ }^{10,11} \mathrm{drug}$ delivery and gene therapy, ${ }^{12-15}$ catalysis, ${ }^{16-18}$ colloidal crystal arrays, ${ }^{19,20}$ the fabrication of superhydrophobic surfaces, ${ }^{21,22}$ and Pickering emulsions ${ }^{23-25}$ ). Environmentally responsive nanometerto micrometer-sized colloidal systems are important in these applications because they offer the possibility to switch macroscopic properties such as hydrophobicity, adhesiveness, and optical properties. A versatile approach to generating responsive colloidal systems is via the surface functionalization of colloidal particles with polymer brushes. Compared to other approaches, polymer brushes offer improved chemical stability and amplification of signal transduction while retaining synthetic flexibility

(10) Gupta, A. K.; Gupta, M. Biomaterials 2005, 26, 3995-4021.

(11) Mornet, S.; Vasseur, S.; Grasset, F.; Duguet, E. J. Mater. Chem. 2004, 14, 2161-2175.

(12) Brigger, I.; Dubernet, C.; Couvreur, P. Adv. Drug Deliverv Rev. 2002, 54 , $631-651$.

(13) Barratt, G. M. Pharm. Sci. Technol. Todav 2000, 3, 163-171.

(14) Jones, M. C.; Leroux, J. C. Eur. J. Pharm. Biopharm. 1999, 48, 101-111.

(15) Das, M.; Zhang, H.; Kumacheva, E. Annu. Rev. Mater. Res. 2006; 36, $117-142$.

(16) Bönnmann, H.; Richards, R. M. Eur. J. Inorg. Chem. 2001, 2455-2480.

(17) Lewis, L. N. Chem. Rev. 1993, 93, 2693-2730.

(18) Ballauff, M. Macromol. Chem. Phvs. 2003, 204, 220-234.

(19) Holtz, J. H.; Asher, S. A. Nature 1997, 389, 829-832.

(20) Chen, Y.; Gautrot, J. E.; Li, Z.; Zhu, X. X. Soft Matter 2007, 3, 571-579.

(21) Motornov, M.; Sheparovych, R.; Lupitskyy, R.; MacWilliams, E.; Minko, S. Adv. Mater. 2008, 20, 200-205.

(22) Sharma, G.; Mei, Y.; Lu, Y.; Ballauff, M.; Irrgang, T.; Proch, S.; Kempe, R. J. Catal. 2007, 246, 10-14.

(23) He, X.; Ge, X.; Liu, H.; Zhou, H.; Zhang, Z. Colloids Surf., A 2007, 301, $80-84$.

(24) Fujii, S.; Read, E. S.; Binks, B. P.; Armes, S. P. Adv. Mater. 2005, 17, 1014 1018.

(25) Tsuji, S.; Kawaguchi, H. Langmuir 2008, 24, 3300-3305. 
(e.g., chemical structure manipulation). ${ }^{26-28}$ In addition, structural properties such as the grafting density and thickness can be controlled easily, therefore providing a greater degree of control. ${ }^{29}$ Motornov et al. $^{21}$ fabricated superhydrophobic surfaces from fumed silica nanoparticles grafted with block copolymer brushes of 4-vinylpyridine and styrene. Aggregates were coated onto silicon wafers to generate superhydrophobic surfaces in which the surface roughness and hence the degree of hydrophobicity can be tuned via $\mathrm{pH}$ and aggregate size. $\mathrm{pH}$-responsive nanoparticles coated with poly(2-vinylpyridine) brushes were exploited in enzymebased logic gates. ${ }^{30}$ Depending on the enzyme input combination and the subsequent biocatalytic reactions, the environmental $\mathrm{pH}$ changed. As a result, particles were switched between aggregated and nonaggregated states and the particle size provided the output signal. Motornov et al. ${ }^{31}$ prepared silica particles coated with a shell of mixed polymer brushes of poly(styrene- $b$-2-vinylpyridine$b$-ethylene oxide) via the grafting-to approach. Because of the $\mathrm{pH}$ responsive poly(2-vinylpyridine) chains, the particles can be used to stabilize both $\mathrm{W} / \mathrm{O}$ and $\mathrm{O} / \mathrm{W}$ emulsions (Pickering emulsions).

In the present study, we generated a responsive colloidal system consisting of silica nanoparticles coated with PMETAC brushes through surface-initiated ATRP. We studied the responsive behavior of these hybrid particles as a function of $\mathrm{ClO}_{4}^{-}$. The effects of the polymer chain length, brush conformation, brush density, and ionic strength on perchlorate-induced particle aggregation were investigated in detail. We showed that supramolecular interactions between PMETAC-based colloidal systems and $\mathrm{ClO}_{4}{ }^{-}$enable the generation and control of Pickering emulsions and studied some of the parameters affecting their stability and homogeneity.

\section{Experimental Section}

Chemicals. 2-(Methacryloyloxy)-ethyl-trimethyl-ammonium chloride (75\%) (METAC), copper(II) chloride, copper(I) bromide, (3-aminopropyl)trimethoxysilane (APTMS), 2-bromoisobutyryl bromide (BIB), propionyl bromide (PB), 2,2'-dipyridyl (bipy), dodecane, and oil blue $\mathrm{N}$ were purchased from Sigma-Aldrich. An ammonia solution $(35 \%)$ and glacial acetic acid were obtained from Fisher Scientific. Triethylamine $\left(\mathrm{Et}_{3} \mathrm{~N}\right)$ was from Alfa Aesar. All chemicals and solvents were of analytical grade and were used as received unless otherwise stated. $\mathrm{Cu}(\mathrm{I}) \mathrm{Br}$ was kept under vacuum. $\mathrm{Et}_{3} \mathrm{~N}$ was distilled from $\mathrm{KOH}$ and stored over molecular sieves (3 $\AA$ grade). Water was purified to a resistivity of $18.2 \mathrm{M} \Omega \cdot \mathrm{cm}$ with a Millipore Synergy system. The ATRP initiator, 2-bromo-2methylpropionic acid 3-trichlorosilanylpropyl ester 1, was synthesized according to the published procedures. ${ }^{32}$ Silicon wafers (100 mm diameter, $\langle 100\rangle$ orientation, one side polished) were supplied by Compart Technology and cleaned in a plasma asher (Emitech, model K1050X) for $10 \mathrm{~min}$ in an air plasma (forward power of $100 \mathrm{~W}$ ). Silica particles (supplied as powder, mean diameters of $320 \mathrm{~nm}$ and $2.5 \mu \mathrm{m}$ ) with $\mathrm{Si}-\mathrm{OH}$ surface functional groups were purchased from Bangs Laboratories.

Deposition of Ester Silane Initiator on Silicon Wafers. ATRP initiator $\mathbf{1}(10 \mu \mathrm{L})$ was added to a crystallization dish

(26) Chen, T.; Ferris, R.; Zhang, J.; Ducker, R.; Zauscher, S. Prog. Polvm. Sci. 2010, 35, 94-112.

(27) Edmondson, S.; Osborne, V. L.; Huck, W. T. S. Chem. Soc. Rev. 2004, 33, $14-22$

(28) Ballauff, M. Prog. Polvm. Sci. 2007, 32, 1135-1151.

(29) Rühe, J. In Polymer Brushes: Synthesis, Characterization, Applications; Advincula, R. C., Brittain, W. J., Caster, K. C., Rühe, J., Eds.; Wiley-VCH: Weinheim, Germany, 2004; pp 1-24.

(30) Motornov, M.; Zhou, J.; Pita, M.; Gopishetty, V.; Tokarev, I.; Katz, E.; Minko, S. Nano Lett. 2008, 8, 2993-2997.

(31) Motornov, M.; Sheparovych, R.; Lupitskyy, R.; MacWilliams, E.; Hoy, O.; Luzinov, I.; Minko, S. Adv. Funct. Mater. 2007, 17, 2307-2314.

(32) Jonas, A. M.; Glinel, K.; Oren, R.; Nysten, B.; Huck, W. T. S. Macromolecules 2007, 40, 4403-4405. containing dry toluene $(30 \mathrm{~mL})$ and $\mathrm{Et}_{3} \mathrm{~N}(50 \mu \mathrm{L})$ and was mixed briefly. A plasma-oxidized silicon wafer was transferred to the dish and immersed in the resulting solution. The dish was covered with aluminum foil and left at room temperature for $18 \mathrm{~h}$. The wafer was washed with acetone, sonicated in acetone for $30 \mathrm{~s}$, rinsed thoroughly with acetone and ethanol, and finally dried under a stream of nitrogen. The ellipsometric thickness of the resulting initiator layer was $0.3-0.6 \mathrm{~nm}$. The initiator-functionalized wafer was kept under nitrogen until use.

Deposition of Ester Silane Initiator on Silica Nanoparticles. Silica nanoparticles $(D \approx 320 \mathrm{~nm}, 50 \mathrm{mg})$ were dispersed in dry toluene by sonication (Branson 3510 ), washed in dry toluene via three centrifugation $(6500 \mathrm{rpm}) /$ redispersion cycles, and finally dispersed in dry toluene $(1 \mathrm{~mL})$. To this dispersion, dry $\mathrm{Et}_{3} \mathrm{~N}$ $(50 \mu \mathrm{L})$ and ATRP initiator $1(10 \mu \mathrm{L})$ were added. The suspension was stirred vigorously at room temperature for $24 \mathrm{~h}$. The resulting particles were collected by centrifugation, washed three times successively with dry toluene and isopropyl alcohol (IPA), and finally redispersed in IPA/water $(4: 1 \mathrm{v} / \mathrm{v})$.

Deposition of Amide Silane Initiator on Silica Nanoparticles. The deposition of amide silane initiator was carried out according to the published procedures. ${ }^{33}$ Silica nanoparticles $(D \approx 320 \mathrm{~nm}$, $50 \mathrm{mg}$ ) were dispersed in ethanol by sonication, washed in ethanol via three centrifugation/redispersion cycles, and dispersed in ethanol $(45 \mathrm{~mL})$. The dispersion was heated to $60{ }^{\circ} \mathrm{C}$. While the dispersion was stirred, aqueous ammonia $(35 \%)(50 \mu \mathrm{L})$ was added, followed by APTMS $(0.4 \mathrm{~mL})$. The reaction was allowed to proceed at $60{ }^{\circ} \mathrm{C}$ for $24 \mathrm{~h}$ with continuous stirring. Subsequently, particles were isolated by centrifugation, washed in anhydrous toluene via three centrifugation/redispersion cycles, and redispersed in anhydrous toluene $(45 \mathrm{~mL})$. Dry pyridine $(0.3 \mathrm{~mL})$ was added to the dispersion and cooled in an ice bath for $30 \mathrm{~min}$. BIB and/or PB (total $2.0 \mathrm{mmol}$ ) was injected dropwise into the vigorously stirred dispersion. The reaction was allowed to proceed at $0{ }^{\circ} \mathrm{C}$ for $1 \mathrm{~h}$ and then at room temperature for $23 \mathrm{~h}$ with continuous stirring. Particles were collected by centrifugation, washed successively three times with dry toluene and IPA, and finally redispersed in IPA/water $(4: 1 \mathrm{v} / \mathrm{v})$.

ATRP on Silicon Wafers. METAC (13.86 g, $50 \mathrm{mmol})$, bipy (328 mg, $2.1 \mathrm{mmol})$, and $\mathrm{Cu}(\mathrm{II}) \mathrm{Cl}_{2}(6.7 \mathrm{mg}, 0.05 \mathrm{mmol})$ were dissolved in IPA/water $(4: 1 \mathrm{v} / \mathrm{v})(20 \mathrm{~mL})$. The resulting solution was degassed via nitrogen bubbling for $30 \mathrm{~min}$ with stirring. $\mathrm{Cu}(\mathrm{I}) \mathrm{Br}(143 \mathrm{mg}, 1 \mathrm{mmol})$ was added, and the resulting solution was degassed for another $30 \mathrm{~min}$. Initiator-coated silicon chips $\left(1 \times 1 \mathrm{~cm}^{2}\right.$ each, cut from an initiator-coated silicon wafer) were sealed in reaction vessels and degassed via four high-vacuum/ nitrogen cycles. Subsequently, the solution was transferred to the vessels $(5 \mathrm{~mL} /$ vessel). Polymerization was allowed to proceed under nitrogen at room temperature for $15 \mathrm{~min}$ to $2 \mathrm{~h}$. The substrates were rinsed thoroughly with water and ethanol and finally dried under a stream of nitrogen.

ATRP on Silica Nanoparticles. Polymerization solutions were prepared as above by dissolving METAC, bipy, $\mathrm{Cu}(\mathrm{I}) \mathrm{Br}$, and $\mathrm{Cu}(\mathrm{II}) \mathrm{Cl}_{2}$ in half of the total polymerization solvent. Initiator-coated silica nanoparticles were dispersed in the other half of the solvent while being continuously stirred and degassed with nitrogen for $30 \mathrm{~min}$. Particles were then injected into the polymerization solution and left at room temperature with continuous stirring and degassing for $5 \mathrm{~min}$ to $24 \mathrm{~h}$. (For the $24 \mathrm{~h}$ sample, $27.7 \mathrm{~g}$ $(100 \mathrm{mmol})$ of METAC and $30 \mathrm{~mL}$ of IPA/water $(4: 1 \mathrm{v} / \mathrm{v})$ were used.) To terminate the polymerization, the dispersion was diluted and compressed air was bubbled through the resulting mixture until its color changed from dark brown to blue-green. Particles were recovered via centrifugation, washed with water and subsequently with ethanol (three times each), centrifuged, and dried under high vacuum at room temperature. This afforded a white powder.

(33) Wu, T.; Zhang, Y.; Wang, X.; Liu, S. Chem. Mater. 2008, 20, 101-109. 
Scheme 1. Illustration of Initiator Deposition and the Formation of PMETAC Brushes ${ }^{a}$

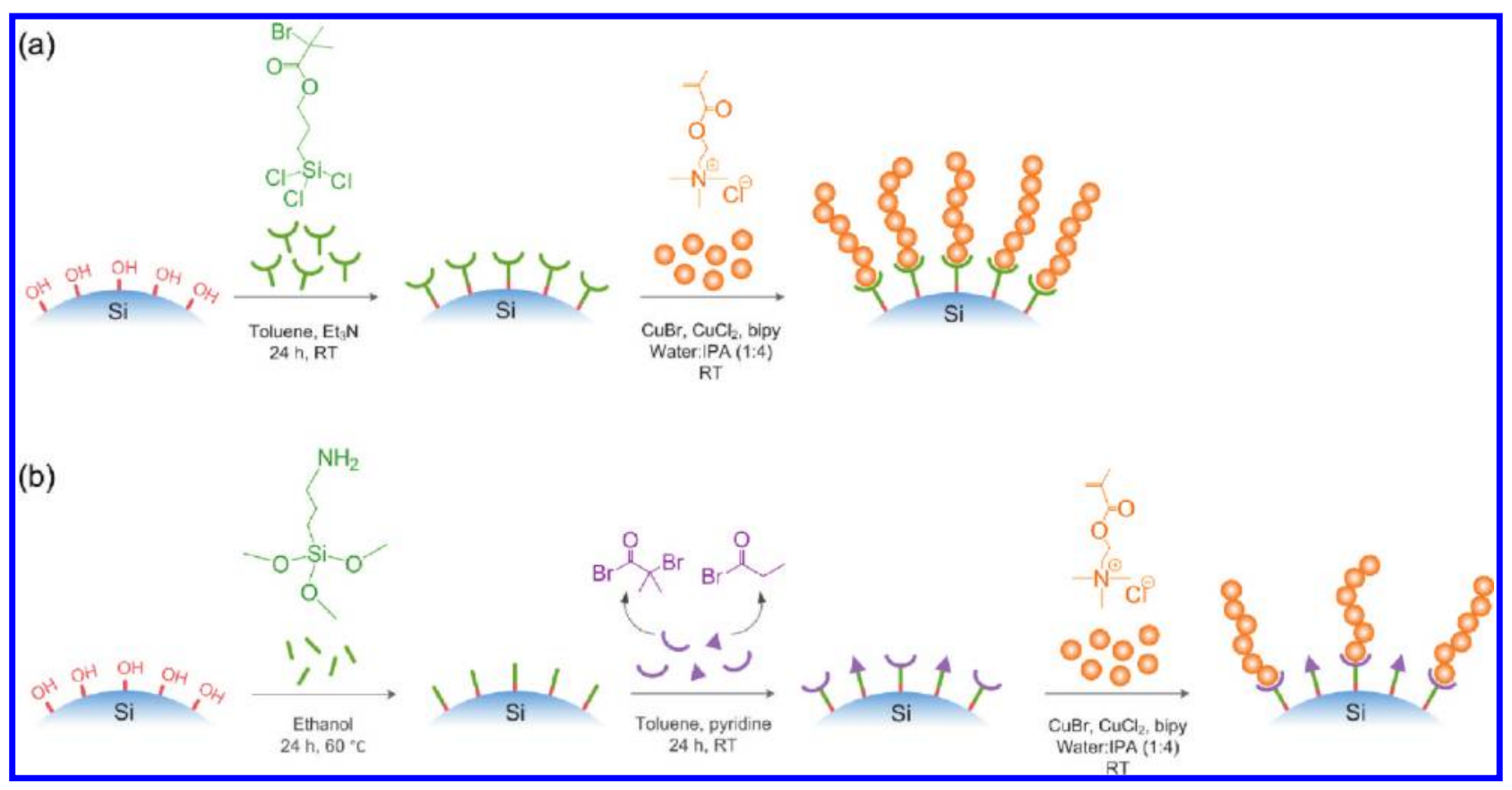

${ }^{a}$ (a) Ester initiator and (b) amide initiator (used to obtain various initiator densities).

Characterization. FT-IR spectroscopy was carried out by using a Perkin-Elmer Spectrum 100 spectrometer. Spectra were taken at a resolution of $4 \mathrm{~cm}^{-1}$ with a total of 128 scans per run. TGA was performed in nitrogen using a TA Instruments Q500 analyzer. Samples were heated from room temperature to $1000^{\circ} \mathrm{C}$ at a heating rate of $10^{\circ} \mathrm{C} / \mathrm{min}$. TEM was conducted on a FEI Philips Tecnai 20 electron microscope at an acceleration voltage of $200 \mathrm{kV}$. Each TEM sample was prepared by depositing a drop of a nanoparticle dispersion $(5 \mu \mathrm{L})$ on a thin carbon film coated onto a $3 \mathrm{~mm} 400$-mesh copper grid (Agar Scientific) and allowing the dispersion to dry completely at room temperature. Polymer film thicknesses on silicon wafers were measured on an $\alpha-S E$ spectroscopic ellipsometer (J. A. Woollam) at an incident angle of $70^{\circ}$. To measure the polymer thickness and swelling in liquids, samples were placed in a home-built liquid cell fitted with quartz windows. Contact angle goniometry was carried out at room temperature by using deionized water and a home-built stage with a computer-controlled microsyringe. The distance between samples and the microsyringe was about $1 \mathrm{~mm}$. Images were taken using a digital camera once infusion was complete. The hydrodynamic particle diameter $\left(D_{\mathrm{H}}\right)$ and the zeta potential of the particles were determined via DLS by using Malvern Zetasizer Nano ZS fitted with a $633 \mathrm{~nm}$ red laser. For DLS, the scatteredlight intensity was measured at a scattering angle of $173^{\circ} . D_{\mathrm{H}}$ was determined according to the Stokes-Einstein equation

$$
D_{\mathrm{H}}=\frac{k T}{f}=\frac{k T}{3 \pi \eta D}
$$

where $k$ is the Boltzmann constant, $f$ is the particle frictional coefficient, $\eta$ is the solvent viscosity, $T$ is the absolute temperature, and $D$ is the translational diffusion coefficient. Particles were assumed to be spherical, monodisperse, and noninteracting. The zeta potential $(\xi)$ of the particles was estimated from the electrophoretic mobility $(u)$ as in the Helmholtz-Smoluchowski equation

$$
u=\epsilon \frac{\xi}{\eta}
$$

where $\eta$ and $\epsilon$ are the viscosity and the dielectric constant of the medium, respectively. For DLS measurement, samples were prepared by dispersing particles in deionized water such that they appeared to be slightly turbid, followed by sonication for $5 \mathrm{~min}$. $\mathrm{NaCl}$ solution was subsequently added to a final concentration of $1 \mathrm{mM}$ to eliminate long-range charge interactions. Three consecutive measurements per sample were carried out at $25^{\circ} \mathrm{C}$ over 10-15 min. Zeta-potential measurements were carried out in deionized water. To investigate the effects of salts on interparticle interactions, particles were first sonicated in deionized water and subsequently salt solutions of known concentrations were added. The resulting dispersion was mixed. For ellipsometry, contact angle, DLS, and zeta-potential measurements, at least three samples were measured and a minimum of three measurements per sample were performed. Measurements are given as averages \pm standard deviations. PMETAC-coated silica particles $(2.5 \mu \mathrm{m}$ silica core particles) were imaged by bright field optical microscopy using a Leica DMI4000 microscope.

Pickering Emulsions. For each sample, PMETAC-coated particles $(0.5-2 \mathrm{mg})$ were dispersed in deionized water $(0.25 \mathrm{~mL})$ and sonicated for $10 \mathrm{~min}$. Dodecane $(0.6 \mathrm{~mL})$ was added to the dispersion and shaken vigorously. $\mathrm{A} \mathrm{NaClO}_{4}$ or $\mathrm{NaCl}$ solution was added to the dispersion to produce the intended final aqueous concentration (1:2 aqueous/oil v/v) and mixed well. The formation of an emulsion was followed before and after the addition of salt (up to $60 \mathrm{~h}$ ). Dodecane was dyed with the oleophilic dye oil blue $\mathrm{N}$ to identify the oil phase in an emulsion, and color emulsion images were taken with a Nikon Eclipse ME600 microscope (10× magnification). For emulsion droplet size analysis, emulsion images were obtained via an Olympus IX71 microscope ( $2 \times$ magnification) $30 \mathrm{~min}$ after an emulsion was formed, and a total of 570-580 droplets were analyzed for each sample. Particle size statistical analysis was performed via one-factor ANOVA and Tukey's test for posthoc analysis. The significance was determined by $P<0.001$. The droplet size was expressed as the average \pm the standard error. The emulsion volume was obtained by subtracting the volumes of single phases (oil and aqueous) from the initial total volume.

\section{Results and Discussion}

Growth of PMETAC Brushes on Silica Nanoparticles. PMETAC brushes were grown on the surface of initiatorfunctionalized silica nanoparticles $(D \approx 320 \mathrm{~nm})$ via aqueous 


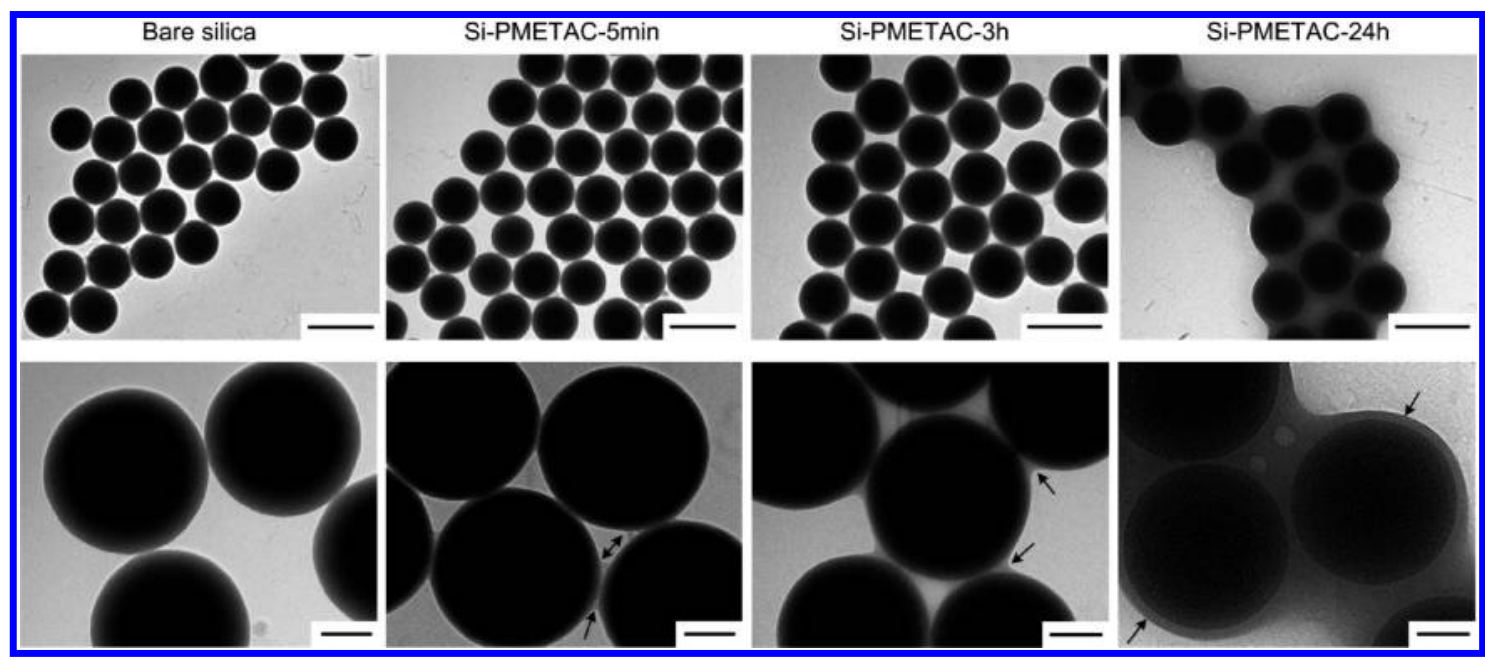

Figure 1. TEM images of bare and PMETAC brush-grafted silica nanoparticles. Polymerization time: 5 min (Si-PMETAC-5 min), 3 h (Si-PMETAC-3 h), and $24 \mathrm{~h}$ (Si-PMETAC-24 h). (Scale bars: top row, $500 \mathrm{~nm}$; bottom row, $100 \mathrm{~nm}$ ). Arrows show PMETAC coatings.

ATRP (Scheme 1a). Several methods were tested to determine the ideal conditions for the deposition of initiator 1 on a silica surface (SI, Figure S1). Anhydrous toluene and $\mathrm{Et}_{3} \mathrm{~N}$ afforded denser and more homogeneous brushes, and this method was used for the rest of this study. Figure 1 shows TEM images of bare and hybrid silica nanoparticles. These images show a gradual increase in the PMETAC coating (in light gray in Figure 1) as the polymerization time increased.

The presence of grafted polymer brushes on silica nanoparticles was further confirmed by FT-IR spectroscopy (SI, Figure S4). The spectra of initiator-functionalized nanoparticles show characteristic peaks at 1382 and $1406 \mathrm{~cm}^{-1}$ corresponding to $\mathrm{C}-\mathrm{H}$ deformation. ${ }^{33,34}$ The spectra of PMETAC brush-grafted particles show distinct peaks for $\mathrm{C}=\mathrm{O}$ stretching $\left(1725 \mathrm{~cm}^{-1}\right)$ and $\mathrm{C}-\mathrm{H}$ bending $\left(1480 \mathrm{~cm}^{-1}\right)$. The $\mathrm{C}-\mathrm{N}^{+}$stretching band $\left(\sim 1260 \mathrm{~cm}^{-1}\right)^{3}$ that is characteristic of quaternary ammonium overlaps with the broad $\mathrm{Si}-\mathrm{O}$ stretching band. An intense, broad peak at $3389 \mathrm{~cm}^{-1}$ is associated with the presence of water molecules in the sample, consistent with the charged structure of PMETAC and its expected hygroscopic behavior.

The thickness of the PMETAC coating was estimated via TGA measurements (SI, Figure S5). The weight loss in TGA traces is attributed to the pyrolysis of organic contents, and residues are assumed to be noncombustible silica. According to the difference in weight loss between initiator-coated and bare silica particles at $800{ }^{\circ} \mathrm{C}(2.2 \mathrm{wt} \%)$, the density of the ATRP initiator immobilized on particles is estimated to be $\sim 7.6$ initiator $/ \mathrm{nm}^{2}$. (See SI for the calculation.) This value agrees with those reported in the literature $^{35}$ for silica particles of similar diameter $(300 \mathrm{~nm})$. It is generally accepted that the surface hydroxyl density of silica is $\sim 5 \mathrm{OH} / \mathrm{nm}^{2}{ }^{36}$ Hence, a dense initiator layer was successfully deposited on silica particles, consistent with results obtained from the deposition of initiators on silicon wafers. The polymer content of hybrid particles was determined with reference to initiatorcoated particles at $800^{\circ} \mathrm{C}$ and was used to calculate the polymer dry thickness. (See SI for the calculation.) The polymer dry thickness on silica nanoparticles increased from 3 to $80 \mathrm{~nm}$ for polymerization times ranging from $5 \mathrm{~min}$ to $24 \mathrm{~h}$ (Table 1).

(34) Socrates, G. Infrared and Raman Characteristic Group Frequencies: Tables and Charts, 3rd ed.; Wiley: Chichester, U.K., 2001.

(35) Perruchot, C.; Khan, M. A.; Kamitsi, A.; Armes, S. P.; Watts, J. F.; Von Werne, T.; Patten, T. E. Eur. Polvm. J. 2004, 40, 2129-2141.

(36) Von Werne, T.; Patten, T. E. J. Am. Chem. Soc. 1999, 121, 7409-7410.
Responsive Behaviors of PMETAC-Coated Silica Nanoparticles. The responsiveness of PMETAC as a function of perchlorate ions was confirmed via ellipsometry on silicon wafers (SI, Figure S2 and Table S2). Subsequently, interactions between PMETAC-modified particles were studied in response to $\mathrm{ClO}_{4}{ }^{-}$ exposure via DLS. As depicted in Figure 2a, upon addition of $5 \mathrm{mM} \mathrm{NaClO}_{4}$, the $D_{\mathrm{H}}$ of all PMETAC-coated particles, regardless of coating thicknesses, decreased abruptly. At intermediate $\mathrm{NaClO}_{4}$ concentrations $(5-25 \mathrm{mM})$, the size of the particles remained remarkably constant, even after long periods of time (34 h) (SI, Figure S6), indicating that although the PMETAC brushes had collapsed, colloidal dispersions remained stable. Aggregation occurred at higher $\mathrm{NaClO}_{4}$ concentrations and was thickness-dependent. (It occurred at 25, 20, and $17.5 \mathrm{mM}$ $\mathrm{NaClO}_{4}$ for Si-PMETAC-5 min, Si-PMETAC-3 h, and Si-PMETAC-24 h, respectively.) Above $25 \mathrm{mM} \mathrm{NaClO}_{4}$, the size of the aggregates that formed could not be measured precisely because they were beyond the upper range of our instrument (i.e., $6 \mu \mathrm{m})$. For all samples, aggregation was accompanied by sedimentation. Optical microscopy images (Figure 3a-c) show that PMETAC-coated silica particles $(2.5 \mu \mathrm{m}$ silica core particles) remained well dispersed in low $\mathrm{NaClO}_{4}$ concentrations and formed large aggregates at increased $\mathrm{NaClO}_{4}$ concentrations.

Zeta-potential profiles of PMETAC-modified particles as a function of $\mathrm{NaClO}_{4}$ concentration are shown in Figure 2b. Large zeta potentials indicate high electrostatic repulsive forces between particles and hence a stable dispersion. The zeta potential of particles coated with PMETAC brushes grown for 5 min to $24 \mathrm{~h}$ decreased from 53 to $38 \mathrm{mV}$ respectively after exposure to $10 \mathrm{mM}$ $\mathrm{NaClO}_{4}$. Hence, in the collapsed intermediate state, electrostatic repulsion is stronger for shorter PMETAC brushes and these particles were more stable than their longer PMETAC homologues (destabilization triggered at 25 instead of $17.5 \mathrm{mM} \mathrm{NaClO}_{4}$ respectively). The zeta-potential profiles that were measured do not give evidence of dramatic changes in the particle surface charge after brush collapse and instead suggest the formation of increasingly unstable colloidal systems at elevated $\mathrm{NaClO}_{4}$ concentrations.

To elucidate the mechanism by which the aggregation of PMETAC-coated particles was triggered, we studied the effects of (1) brush conformational changes, (2) initiator density, and (3) electrostatic charge shielding on interparticle interactions. 


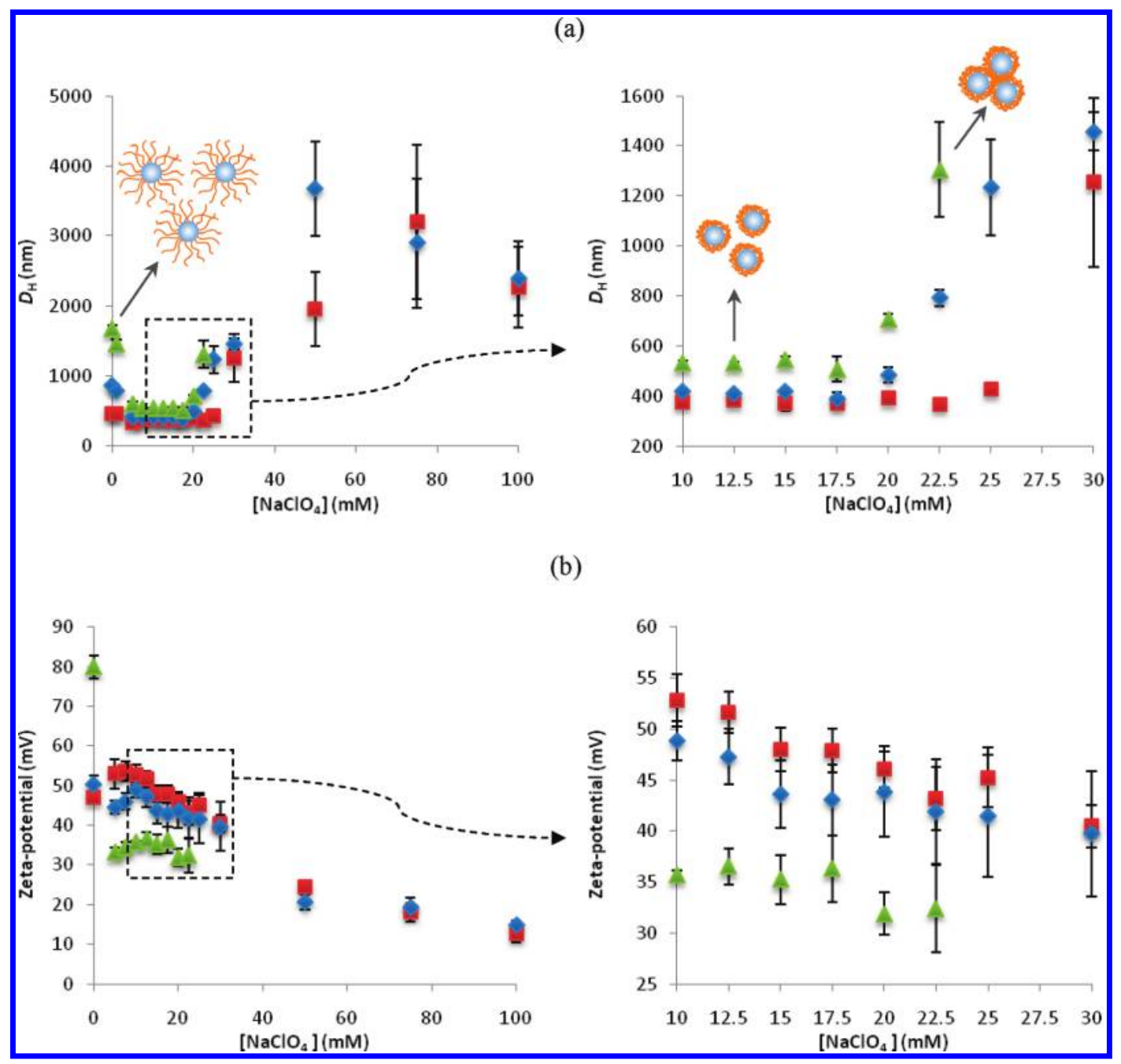

Figure 2. Responsive behavior of PMETAC-coated silica nanoparticles upon exposure to $\mathrm{NaClO}_{4}$. (a) $D_{\mathrm{H}}$ and (b) zeta potential of

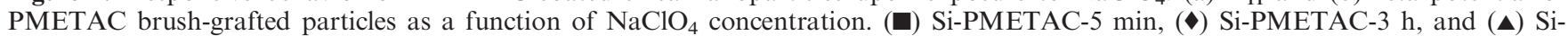
PMETAC-24 h.

Table 1. PMETAC Content and Dry Thickness on Silica Nanoparticles

\begin{tabular}{lccc}
\hline \multicolumn{1}{c}{ sample } & $\begin{array}{c}\text { polymerization } \\
\text { time }\end{array}$ & $\begin{array}{c}\text { polymer } \\
\text { content } \\
(\%)^{a}\end{array}$ & $\begin{array}{c}\text { polymer dry } \\
\text { thickness } \\
(\mathrm{nm})^{a}\end{array}$ \\
\hline Si-PMETAC-5 min & $5 \mathrm{~min}$ & 3.2 & 3.2 \\
Si-PMETAC-3 $\mathrm{h}$ & $3 \mathrm{~h}$ & 11.0 & 11.8 \\
Si-PMETAC-24 h & $\begin{array}{c}24 \mathrm{~h} \\
{ }^{a} \text { Calculated on the }\end{array}$ & $\begin{array}{c}52.1 \\
80.1\end{array}$
\end{tabular}

basis of TGA weight loss with reference to initiator-coated silica particles.

Effect of Brush Conformational Changes and Grafting Density on Particle Aggregation. The responsiveness of PMETAC particles was found to be independent of brush conformation. We hypothesized that particle aggregation may arise from the conformational change (collapse) in PMETAC brushes. In this case, solution ellipsometry should provide evidence of this mechanism and it should be markedly sensitive to the brush density (which is known to affect the brush conformation strongly). ${ }^{29}$ PMETAC brushes grown on silicon wafers were sequentially exposed to deionized water and $\mathrm{NaClO}_{4}$ solutions of increasing concentrations, and their thickness was followed by ellipsometry. For both thin $(10 \mathrm{~nm})$ and thick $(60-80 \mathrm{~nm})$ brushes, full collapse was observed even at $5 \mathrm{mM} \mathrm{NaClO}_{4}$ and subsequent increases in concentration did not result in further changes in brush swelling (Figure 4a) or water content. (The refractive index extrapolated

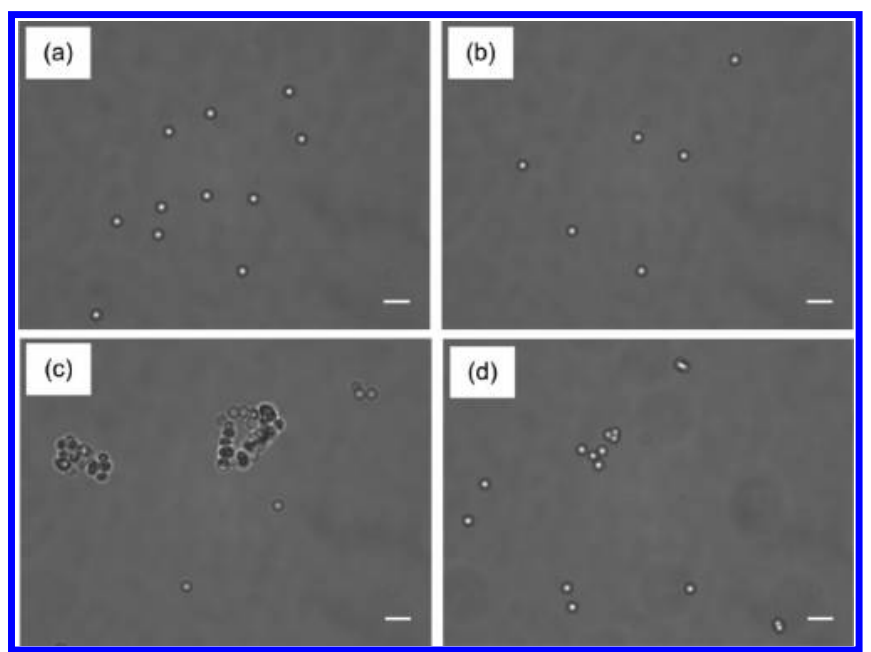

Figure 3. Optical microscopy images of PMETAC-coated silica particles $(2.5 \mu \mathrm{m}$ silica core particles) in (a) water, (b) $10 \mathrm{mM}$ $\mathrm{NaClO}_{4}$, (c) $50 \mathrm{mM} \mathrm{NaClO}_{4}$, and (d) $10 \mathrm{mM} \mathrm{NaClO}$ and $50 \mathrm{mM}$ $\mathrm{NaCl}(10 \mu \mathrm{m}$ scale bars). ATRP was carried out for $3 \mathrm{~h}$ according to the standard procedure for $320 \mathrm{~nm}$ silica particles.

from these measurements remained relatively constant without a marked transition; data are not shown.) 


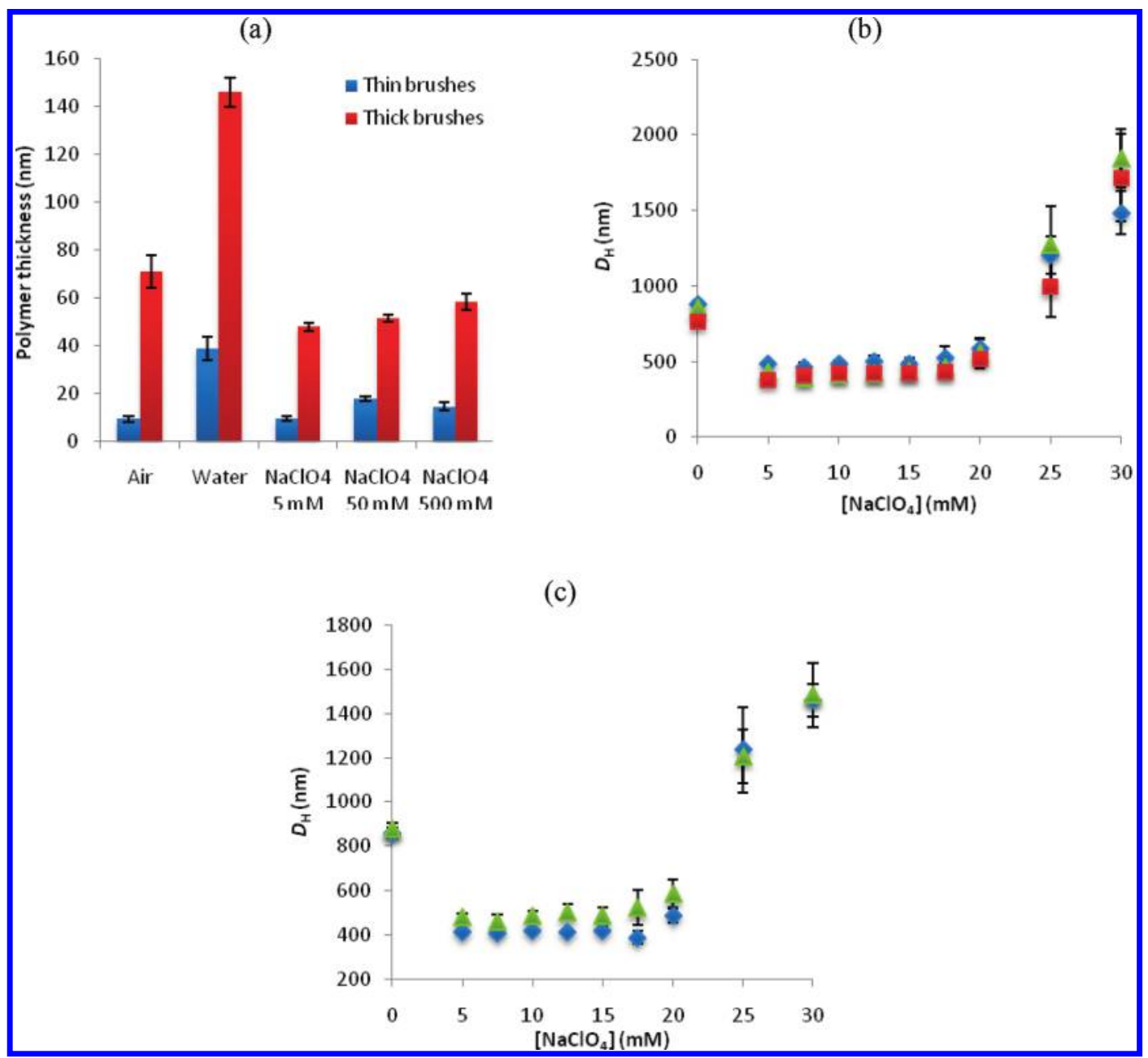

Figure 4. (a) Ellipsometric thickness of PMETAC brushes on silicon wafers measured in air, then in water, and finally in $\mathrm{NaClO}_{4}$. (b) $D_{\mathrm{H}}$ of

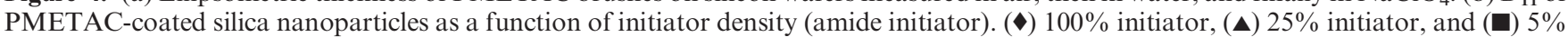
initiator. (c) $D_{\mathrm{H}}$ profiles of PMETAC-coated silica nanoparticles as a function of $\mathrm{NaClO}_{4}$ concentration and initiator type. ( $)$ Amide initiator and $(\mathbf{\Delta})$ ester initiator.

To examine the effect of brush conformation on colloidal responsiveness, particles were coated with brushes of decreasing density (100, 25, and 5\% initiator densities) synthesized following the procedure reported by $\mathrm{Wu}$ et $\mathrm{al}^{33}$ (Scheme $1 \mathrm{~b}$ ). ATRP of METAC was carried out for $3 \mathrm{~h}$ in all three cases, and FT-IR spectra of the resulting particles (SI, Figure S7) showed characteristic peaks of PMETAC brushes. TEM images of particles with 100 and 5\% initiator are presented in the SI (Figure S8) and confirm that both types of particles are coated with PMETAC. DLS measurements showed that, for all brush densities, particles grafted with PMETAC exhibited similar $D_{\mathrm{H}}$ values $(915-1037 \mathrm{~nm})$, in agreement with the identical polymerization times (Figure 4b). In addition, the brush density did not have any effect on particle aggregation and destabilization, which occurred at $20 \mathrm{mM} \mathrm{NaClO}_{4}$ and higher concentrations in all three cases. Finally, particle aggregation was not affected by the type of silane initiator used: PMETAC-modified particles based on amide and ester initiators exhibited analogous aggregation profiles (Figure 4c).

Effect of Electrostatic Shielding on Particle Aggregation. According to the classical Derjaguin-Landau-Verwey-Overbeek (DLVO) theory, colloidal stability in polar solutions is governed by two independent opposing forces: attractive van der Waals

(37) Dougherty, G. M.; Rose, K. A.; Tok, J. B. H.; Pannu, S. S.; Chuang, F. Y. S.; Sha, M. Y.; Chakarova, G.; Penn, S. G. Electrophoresis 2008, 29, 1131-1139. interactions and repulsive electrostatic forces. ${ }^{37}$ The energy barrier resulting from the electrostatic repulsive forces prevents two particles from approaching each other. If this energy is overcome and particles collide, then attractive forces result in particle aggregation and destabilization of the colloidal dispersion. ${ }^{38}$

The impact of electrostatic shielding on aggregation was studied by immersing PMETAC-coated particles in mixed $\mathrm{NaClO}_{4}$ and $\mathrm{NaCl}$ solutions: the concentration of $\mathrm{NaClO}_{4}$ was kept constant $(10 \mathrm{mM})$, and that of $\mathrm{NaCl}$ was gradually increased. This resulted in an abrupt increase $(501-846 \mathrm{~nm})$ in the particle $D_{\mathrm{H}}$ near $20 \mathrm{mM}$ $\mathrm{NaCl}$ (Figure 5a). This behavior contrasted with that of particles in pure $\mathrm{NaCl}$ solutions (no $\mathrm{NaClO}_{4}$ ), which showed a gradual decrease in $D_{\mathrm{H}}$ with increasing $\mathrm{NaCl}$ concentration, indicative of electrostatic shielding effects (no particle aggregation observed) (Figure 5d). Experiments conducted on flat silicon surfaces coated with PMETAC brushes, followed by ellipsometry, did not show any reswelling when $10 \mathrm{mM} \mathrm{NaClO}$-collapsed brushes were exposed to increasing concentrations of $\mathrm{NaCl}$ (Figure 5c). The optical microscopy image (Figure 3d) confirms the formation of small aggregates of PMETAC-coated particles $(2.5 \mu \mathrm{m}$ silica core particles) in a solution of $10 \mathrm{mM} \mathrm{NaClO}_{4}$ and $50 \mathrm{mM} \mathrm{NaCl}$ (although these aggregates are much smaller than those observed

(38) Liang, Y.; Hilal, N.; Langston, P.; Starov, V. Adv. Colloid Interface Sci. 2007, 134-135, 151-166. 


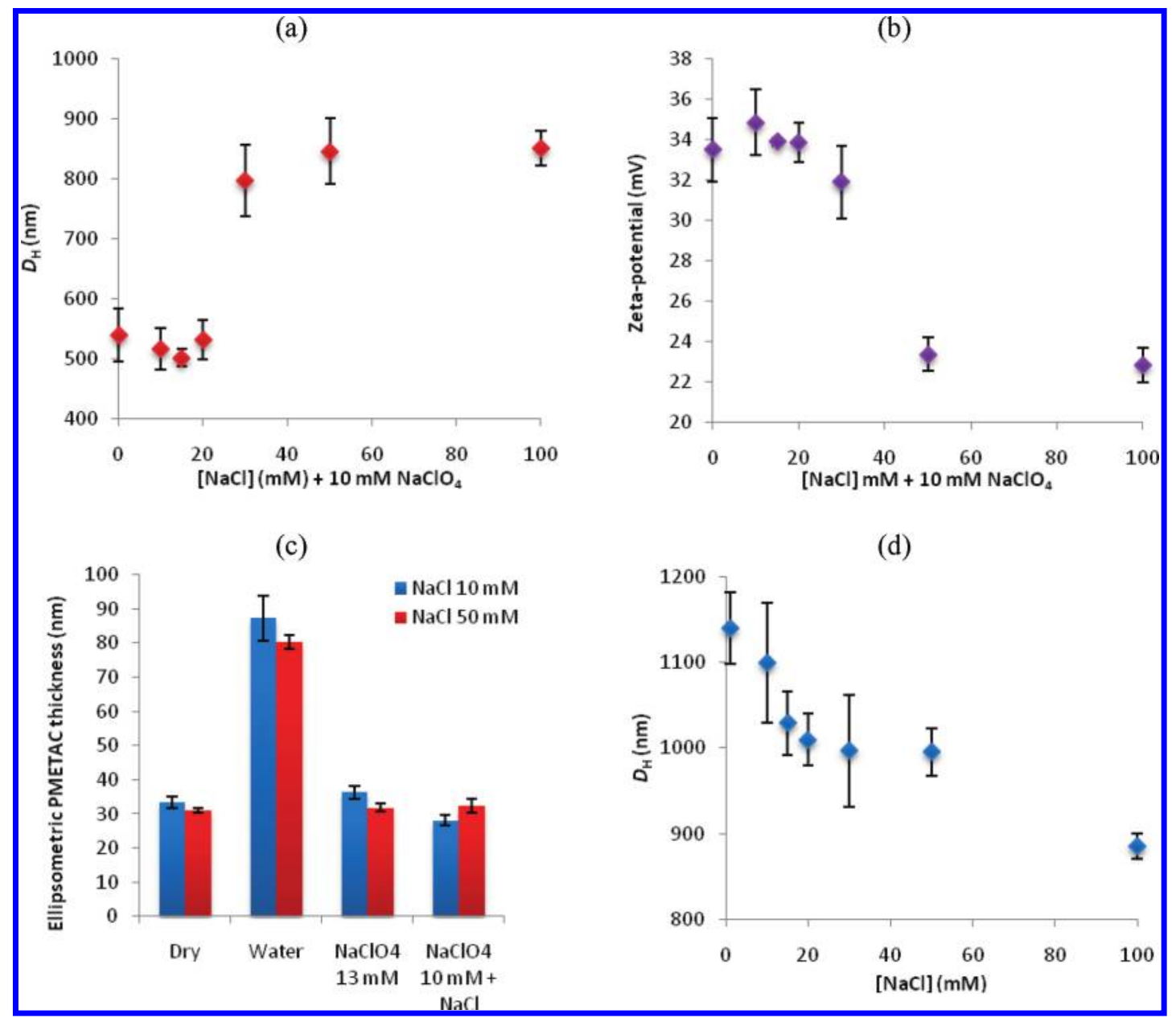

Figure 5. (a) $D_{\mathrm{H}}$ and (b) zeta potential of PMETAC-coated silica nanoparticles as a function of $\mathrm{NaCl}$ concentration. (c) Evolution of the ellipsometric thickness of PMETAC brushes when incubated in different solutions, in sequential order. $\mathrm{NaCl}_{\text {was }}$ added to $\mathrm{NaClO}_{4}$ solutions containing substrates. (d) $D_{\mathrm{H}}$ of PMETAC-coated silica nanoparticles at increasing $\mathrm{NaCl}$ concentrations.

in pure perchlorate solutions). The zeta potential of PMETACcoated particles was monitored upon exposure to mixed $\mathrm{NaClO}_{4}$ $(10 \mathrm{mM})$ and $\mathrm{NaCl}$ solutions (Figure $5 \mathrm{~b}$ ): it was found to decrease substantially when the $\mathrm{NaCl}$ concentration was raised from 30 to $50 \mathrm{mM}$, as in pure $\mathrm{NaClO}_{4}$ experiments (Figure 2b). These experiments strongly suggest that particle aggregation occurs when electrostatic repulsive forces decrease to a critical point as a result of the increased ionic strength of the solution. The smaller aggregate size of the particles exposed to mixed $\mathrm{ClO}_{4}^{-} / \mathrm{Cl}^{-}$ solutions (Figure 5a) compared to pure $\mathrm{ClO}_{4}{ }^{-}$(Figure 2a) may arise from the nature of the interaction of $\mathrm{Cl}^{-}$and $\mathrm{ClO}_{4}^{-}$ions with PMETAC brushes: whereas the former is involved only in electrostatic shielding, the latter also results in increased hydrophobicity. The water contact angle of PMETAC-coated silicon wafers treated with $\mathrm{NaClO}_{4}$ exhibited an increase of $40^{\circ}$ (to $59^{\circ}$ ) compared to those treated with $\mathrm{NaCl}\left(19^{\circ}\right)$, which is consistent with the reported values. ${ }^{2,3}$ Related to this, Azzaroni et al. ${ }^{2}$ showed that the wettability of PMETAC brushes follows the counterion lipophilicity scale and decreases in the order of $\mathrm{PO}_{4}{ }^{3-}$ $>\mathrm{Cl}^{-}>\mathrm{SCN}^{-}>\mathrm{ClO}_{4}^{-}$(water contact angles of $19,35,65$, and $79^{\circ}$, respectively).

Pickering Emulsion Controlled by an Ion-Specific Trigger. The perchlorate ion-specific responsiveness of PMETAC-coated silica nanoparticles and their associated change in surface properties were exploited for the generation and control of Pickering emulsions. As demonstrated in Figure 6, when aqueous dispersions of PMETAC-coated particles were vigorously mixed with dodecane, no stable emulsion formed, even in the presence of $10 \mathrm{mM} \mathrm{NaClO}$, a concentration at which PMETAC brushes were collapsed. However, when the perchlorate concentration was increased to $40 \mathrm{mM}$, the concentration at which PMETACcoated particles aggregated, an emulsion quickly formed upon shaking and remained stable for $60 \mathrm{~h}$. The stability of the emulsion was probably due to the fact that $\mathrm{ClO}_{4}{ }^{-}$ions decreased the osmotic pressure throughout the brush and hence prevented the rediffusion of water molecules. ${ }^{39}$ Therefore, $\mathrm{ClO}_{4}{ }^{-}$-treated brushes did not reswell and remained collapsed in water (SI, Figure S3). In addition, Ashby and Bink ${ }^{40}$ suggested that the energy of attachment of flocculated particles at the emulsion/water interface is greater than that of individual particles because of their apparent size, hence preventing the desorption of these particles upon collision between emulsion droplets. Microscopy images of this emulsion (using oil blue $\mathrm{N}$ to identify the oil phase) clearly showed its oil-in-water structure. In contrast, when $\mathrm{NaCl}$ was used instead of $\mathrm{NaClO}_{4}$, no emulsion was formed, even at higher ionic strength (Figure 6b,c). These results are in good agreement with the responsive behaviors of PMETAC-coated particles as a function of $\mathrm{NaClO}_{4}$ concentration, as discussed earlier.

The effect of the PMETAC-modified particle concentration on the emulsion volume fraction and droplet size was studied.

(39) Moya, S. E.; Azzaroni, O.; Kelby, T.; Donath, E.; Huck, W. T. S. J. Phvs. Chem. B 2007, 111, 7034-7040.

(40) Ashby, N. P.; Binks, B. P. Phvs. Chem. Chem. Phvs. 2000, 2, 5640-5646. 


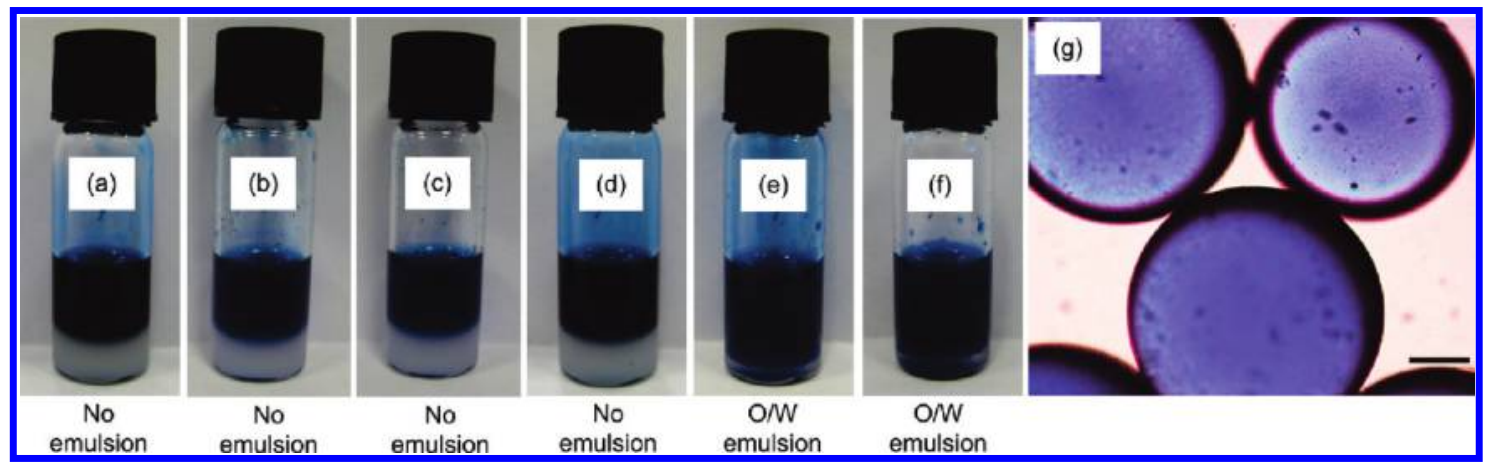

Figure 6. Pickering emulsions prepared using PMETAC-coated particles $\left(D_{\mathrm{H}}=924 \pm 19 \mathrm{~nm}, 0.8\right.$ wt \% particles). Aqueous phase: (a) deionized water after $5 \mathrm{~min}$, (b) $18.5 \mathrm{mM} \mathrm{NaCl}$ after $5 \mathrm{~min}$, (c) $100 \mathrm{mM} \mathrm{NaCl}$ after $5 \mathrm{~min}$, (d) $10 \mathrm{mM} \mathrm{NaClO} 4$ after $5 \mathrm{~min}$, (e) $40 \mathrm{mM}$ $\mathrm{NaClO}_{4}$ after $5 \mathrm{~min}$, and (f) $40 \mathrm{mM} \mathrm{NaClO} 4$ after $60 \mathrm{~h}$. (g) Optical microscopy image of the emulsion formed in part e (50 $\mu \mathrm{m}$ scale bar). The organic phase was dyed blue using oil-soluble oil blue $\mathrm{N}$ to allow the identification of oil and aqueous phases.

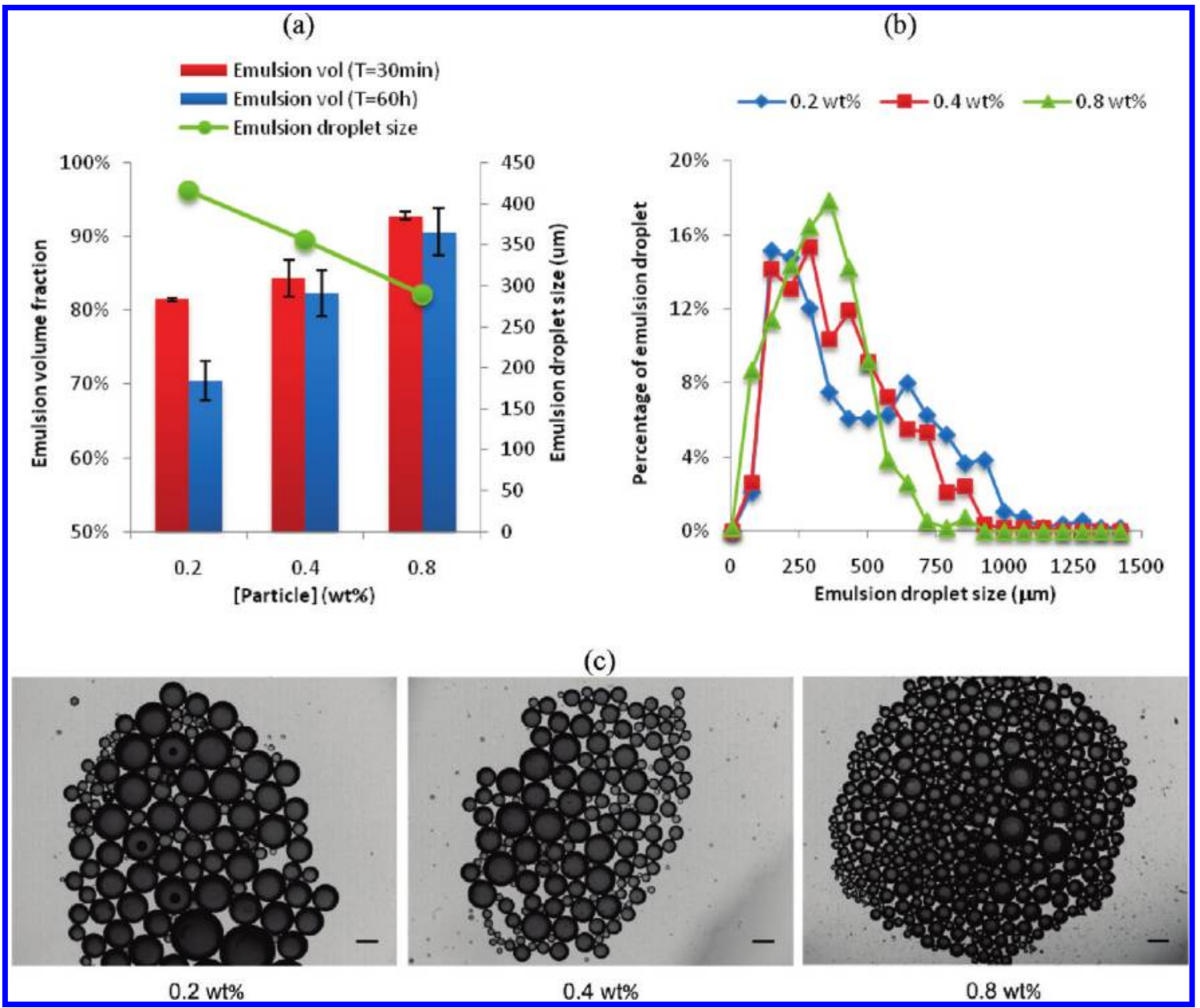

Figure 7. Effect of the concentration of PMETAC-coated silica nanoparticles. (a) Emulsion volume and droplet size, (b) emulsion droplet size distribution, and (c) optical microscopy images of emulsions as a function of the PMETAC-coated particle concentration (500 $u$ m scale bars). Particles with $D_{\mathrm{H}}=1139 \pm 22 \mathrm{~nm}$ were used, and the final $\mathrm{NaClO}_{4}$ concentration was $40 \mathrm{mM}$ in all cases. All emulsions exhibited the oil-in-water structure.

Increasing the particle concentration resulted in smaller droplets, a higher emulsion volume, and increased stability, as shown in Figure 7. The emulsion volume increased with the particle concentration from $81 \%(0.2 \mathrm{wt} \%)$ to $93 \%(0.8 \mathrm{wt} \%)$. The emulsions generated using 0.8 and $0.4 \mathrm{wt} \%$ particles remained stable after $60 \mathrm{~h}$ at room temperature, showing only a $2 \%$ reduction in emulsion volume. In comparison, the emulsion prepared from $0.2 \mathrm{wt} \%$ particles exhibited a more significant reduction $(11 \mathrm{wt} \%)$ after the same period of time. The average droplet diameter increased from 289 to $416 \mu \mathrm{m}$ for emulsions prepared from 0.8 and 0.2 wt $\%$ particles, respectively, with a shift in droplet size distribution as shown in Figure $7 \mathrm{~b}$.

\section{Conclusions}

Colloidal systems based on cationic PMETAC brushes respond to perchlorate ion exposure by an initial collapse of the brush at low concentrations, followed by electrostatic-shielding-induced aggregation at higher ionic strength. This phenomenon was exploited 
for the generation of stable Pickering emulsions. Given the importance of signal amplification for sensing, the control and understanding of particle aggregation is essential because it may provide a basis for very efficient signal transduction, even at low analyte concentrations. Our work shows that polymer brushes can be used to control particle aggregation and that their responsiveness dictates colloidal stability that is independent of ionic strength, a useful feature for probing systems of biological relevance.

Acknowledgment. We thank Dr. Richard Langford of the Electron Microscopy Suite of the Cavendish Laboratory for TEM training. We also thank Dr. Ron Oren for help with ellipsometry measurements. K.Y.T. thanks Schlumberger for financial support.

Supporting Information Available: Deposition of ATRP initiators on silicon wafers. Responsive behaviors of PMETAC brushes on silicon wafers. $D_{\mathrm{H}}$ profile of PMETACcoated silica nanoparticles in $10 \mathrm{mM} \mathrm{NaClO}_{4}$ as a function of time. FT-IR spectra, TGA spectra, and TEM images of PMETAC-coated silica nanoparticles. Calculation of the ATRP initiator density and polymer dry thickness on silica nanoparticles. This material is available free of charge via the Internet at http://pubs.acs.org. 